\title{
THEORETICAL MODELS OF WELL-BEING: SUBJECTIVE, PSYCHOLOGICAL, SENSE OF LIFE, SELF-ESTEEM AND OPTIMISM
}

\author{
Maria Gabriela Costa Ribeiro ${ }^{1}$
}

Abstract: This work aimed to carry out a literature review on the theoretical models of well-being. Specifically, subjective well-being and psychological well-being, in addition to constructs that also promote quality of life, namely, the meaning of life, self-esteem and optimism. For this, the research was structured to present the definitions around the concept of well-being. Then, it tried to conceptualize, to know the psychological measures and studies that demonstrate the relation between sense of life, self-esteem and optimism. In this way, this review demonstrated how research conceives the phenomena in question, showing that it is not up to a single model to understand healthy psychological adjustment, but based on its multidimensionality observed in different constructs.

Keywords: well-being, sense of life, self-esteem, optimism.

\section{Introduction}

Nos últimos, diferentes pesquisadores na área da Psicologia centram seus estudos pelos efeitos do bem-estar psíquico na vida das pessoas. Esse fenômeno psicológico, o bem-estar, refere-se à combinação de se sentir bem e funcionar de maneira eficaz. O bemestar sustentável não exige que os indivíduos se sintam bem o tempo todo; a experiência de emoções dolorosas (por exemplo, decepção, fracasso, tristeza) é uma parte normal da vida, e ser capaz de gerenciar essas emoções negativas ou dolorosas é essencial para o bem-estar a longo prazo. O bem-estar psicológico é, no entanto, comprometido quando as emoções negativas são extremas ou muito duradouras e interferem na capacidade de uma pessoa de funcionar em sua vida diária (Diener et al., 2017). A ideia de se sentir bem incorpora não apenas as emoções positivas de felicidade e alegria, mas também emoções como confiança, envolvimento e afetividade. $O$ conceito de funcionar de forma eficaz (no sentido psicológico)

\footnotetext{
${ }^{1}$ Professora do Curso de Psicologia da Faculdade Três Marias. Email: mariagabicr@gmail.com ORCID: https://orcid.org/0000-0001-6920-9070
} 
envolve o desenvolvimento do potencial de alguém, tendo algum controle sobre sua vida, tendo um senso de propósito (por exemplo, trabalhar para objetivos valorizados) e vivenciar relacionamentos positivos (Ryan e Deci, 2001). Essa definição vai de encontro ao conceito apresentado pela Organização Mundial da Saúde, em que a saúde mental positiva foi definida como um estado de bemestar em que a pessoa percebe suas próprias habilidades, pode lidar com as tensões normais da vida, pode trabalhar de forma produtiva e frutífera e é capaz de fazer uma contribuição para sua comunidade (OMS, 2001).

$\mathrm{Na}$ Psicologia, existem diferentes modelos teóricos do bem-estar, contudo, há construtos que promovem o ajustamento psicológico saudável do indivíduo. Por exemplo, embora exista diferentes modelos acerca do bem-estar, tais como o bem-estar subjetivo (Diener, 1984) e o bem-estar psicológico (Ryff, 1989), decidiu-se apresentar os conceitos de sentido de vida, autoestima e otimismo como modelos teóricos do bem-estar, uma vez que estes contribuem para a qualidade de vida das pessoas (Kleftaras e Psarra, 2012).

Portanto, esta pesquisa procura realizar uma revisão sobre os diferentes modelos teóricos do bem-estar, além dos construtos de sentido de vida, autoestima e otimismo. Esta revisão resume os achados sobre a conceituação dos construtos, as principais medidas utilizadas e estudos que demonstram a sua relação com o bem-estar.

\section{Literature review}

\subsection{Modelos teóricos do bem-estar}

Bem-estar Subjetivo

O Bem-estar Subjetivo (BES) é um construto psicológico que busca compreender como os indivíduos pensam e sentem sobre o que possuem e os eventos que acontecem em suas vidas (Maddux, 2018). O estudo do bem-estar subjetivo abrange o entendimento de como as pessoas evoluem durante a vida, envolvendo tanto o momento presente como período mais longo (e.g, semanas, meses, anos) sobre as reações emocionais das pessoas, tais como seus julgamentos acerca de sua satisfação com a vida, bem como a satisfação em outros domínios da vida (e.g., casamento, trabalho) (Diener, Oishi e Lucas, 2003).

Entre as décadas de 60 e 70, psicólogos e sociológicos demonstraram o interesse no conhecimento a respeito de fatores que influenciam a qualidade de vida das pessoas (Diener, et al., 2017; Maddux, 2018). Entretanto, o 
entendimento da saúde mental ainda se limitava a procurar o motivo da ausência de sintomas psicopatológicos (e.g., depressão, ansiedade) de modo que carecia estudos sobre o desenvolvimento dos aspectos cognitivos e emocionais, assim como o entendimento da variação e adaptação dos sentimentos individuais (Diener et al., 2003). Neste sentido, Diener (1984) na tentativa de preencher essa lacuna teórica integrou os modelos existentes da época e sistematizou o conceito de bem-estar subjetivo.

É importante ressaltar que apesar da confusão na terminologia, bem-estar subjetivo não é equivalente ao conceito de felicidade, em que este último "é um termo solto e com muitos significados e, portanto, é frequentemente evitado na literatura cientifica" (Diener et al., 2017: 87 ff.). Em contrapartida, o termo bemestar subjetivo se refere para as diversas formas na evolução das experiências pessoais na vida, como afetos positivos, afetos negativos e satisfação com a vida (Diener, 2013).

Os componentes do bem-estar subjetivo são divididos em três dimensões: satisfação com a vida (elemento cognitivo), afetos positivos e afetos negativos (aspectos emocionais). Especificamente, a satisfação com a vida diz respeito a uma avalição cognitiva positiva de forma geral em relação à vida, enquanto os afetos positivos e os afetos negativos indicam a frequência de emoções (Woyciekoski, Stenert e Hutz, 2012). Este modelo representa a perspectiva hedônica do bem-estar, o qual enfoca a bivalência entre prazer e desprazer de modo que há a sobreposição de emoções agradáveis (Giacomoni, 2004).

Desse modo, observa-se o interesse de diversos pesquisadores no estudo do bem-estar subjetivo, uma vez que o aumento deste fenômeno possui valor prático na vida das pessoas (Maddux, 2018). Indivíduos com maior bem-estar subjetivo, apresentam sucesso em diferentes áreas na vida, ou seja, são mais sociais, altruístas e ativos; gostam mais de si e dos outros, apresentam melhor habilidade em resoluções de conflito (Lyubomirsky, King e Diener, 2005), maior pensamento criativo (Eid e Larsen, 2008) e experimentam maior saúde física e longevidade (Diener et al., 2017). Portanto, o entendimento deste construto se apresenta essencial no âmbito da Psicologia dado a sua implicação na promoção do ajustamento psicológico. O próximo subtópico busca apresentar outra variável primordial que contribui na vida das pessoas. 
Bem-estar Psicológico

\section{O Bem-estar Psicológico} proposto Carol Ryff (1989) é um modelo teórico sobre o bem-estar que visa entender de forma mais aprofundada quais são as características essenciais deste construto (Ryff, 2014). A partir das concepções divergentes na Psicologia (e.g., clínica, humanista, existencial, desenvolvimento) no funcionamento positivo do psíquico, Ryff (1989) encontrou pontos que convergiam entre as áreas e que serviram para subsidiar um conceito mais consistente do bem-estar.

O modelo de bem-estar psicológico se refere a perspectiva eudaimônica. Esta concepção, originouse no conceito de eudaimonia utilizado por Aristóteles para compreender a ideia que a "boa vida" envolve verdade e responsabilização pessoal (Ruini e Ryff, 2016). Assim, o conceito de bem-estar nesta perspectiva enfatiza a ideia que o indivíduo exerce plenamente sua capacidade, e consequentemente floresce. O bem-estar ocorre, portanto, na extensão das potencialidades, propósitos de vida e realização de metas, isto é, busca capturar os aspectos centrais do que significa ser humano (Ryff, 2014).

Neste sentido, Ryff (2016) propões seis dimensões, os quais correspondem aos componentes da sua medida, sendo eles: 1) propósito de vida, o grau de significado na vida; 2) autonomia, viver de acordo com suas convicções pessoais; 3) crescimento pessoal, uso de talentos e potenciais pessoal; 4) domínio ambiental, o gerenciamento das situações da vida; 5) relações positivas, intimidade nas conexões sociais; 6) autoaceitação, o conhecimento e aceitação pessoal, incluindo suas limitações.

As dimensões que compõe o bem-estar psicológico se apresentam como um construto que recebe atenção entre os pesquisadores, principalmente com o foco no tratamento clínico (Ryan e Deci, 2001). Por exemplo, BEP é fortemente correlacionado com depressão (Wood e Joseph, 2010), sugerindo que indivíduos que demonstram menor nível no BEP, talvez apresente uma maior tendência de desenvolver desordem patológica (e.g., ansiedade, síndrome do pânico). Além disso, Fava (2016) propôs um modelo psicoterápico denominado "Terapia do Bem-estar", o qual utiliza os fundamentos da terapia cognitivocomportamental, assim como o modelo teórico do bem-estar psicológico no tratamento de sintomatologias. 
Outros modelos teóricos do bem-estar

O modelo do bem-estar na perspectiva hedônica ou eudaimônica aparecem frequentemente como objeto de interesse pelos pesquisadores, sobretudo, após o movimento recente da Psicologia Positiva (Seligman e Csikszentmihalyi, 2000). Entretanto, outros modelos foram propostos para o entendimento deste construto na tentativa de integrar ou sistematizar os pontos convergentes entre eles.

Ryan e Deci (2001), por exemplo, adotaram a perspectiva eudaimônica e focalizaram na integração de três dimensões: autonomia (agir de maneira autônoma ou consensual), competência (perseguir objetivos e valores intrínsecos) e conexão (estar atento e agir com um senso de consciência). Estes autores combinaram o modelo eudaimônica com a teoria da autodeterminação, em que as três dimensões possuem implicações motivacionais de modo que os indivíduos com alto bem-estar eudaimônico tendem a se comportar de forma mais pró-social (Ryan e Deci, 2013).

Outro que adotou os seis componentes do bem-estar psicológico foi Keyes (2006). Este autor sugere a "terceira geração do bem-estar" ao incluir a dimensão social, o qual significa as percepções individuais na qualidade das relações com as outras pessoas, como os vizinhos e a comunidade (Keyes, 2006). Assim, adicionou cinco componentes para representar bem-estar social, a saber: aceitação, atualização, contribuição, coerência e integração.

Por sua vez, Seligman (2011) desenvolveu um modelo em consonância com o movimento da psicologia positiva e teve como objetivo a compreensão de quais são os elementos que as pessoas escolhem para o seu próprio bem. Assim, associado ao conceito de flourishing, o qual diz respeito ao bom funcionamento do estado psicológico, o autor desenvolveu o PERMA (EERSR), que é constituído pelos seguintes componentes: emoção positiva, engajamento, relações, sentido $\mathrm{e}$ realização (Butler e Kern, 2016).

Por fim, o Nested Model proposto por Henriques (2011) buscou integralizar os modelos existentes e construiu uma estrutura acerca do bemestar. O autor mapeou o construto em quatro domínios inter-relacionados, apesar de serem distintos: (a) domínio subjetivo (felicidade e satisfação com a vida); (b) domínio da saúde e funcional (biológico e psicológico do indivíduo); 
c) ambiental (contexto material e social); e (d) domínio dos valores e ideologia (a perspectiva moral e ética externa do observador e avaliador). A combinação desses elementos serviu de base para formar um conceito mais holístico e integrado do bem-estar (Henriques, Kleinman, e Asselin, 2014).

Diante do exposto, observa-se a multiplicidade de modelos teóricos na compreensão do bem-estar. Todavia, independente da teoria utilizada, elas ressaltam principalmente a promoção no ajustamento psicológico saudável dos indivíduos. Neste sentido, outros construtos contribuem no aumento do bem-estar, como o significado existencial, autoestima e otimismo. Portanto, os tópicos subsequentes descreverão, respectivamente o conceito, medidas e correlatos desses construtos.

\subsection{Sentido de Vida}

Definição

Durante a história da Psicologia, observa-se o interesse dos pesquisadores para o entendimento do conceito sentindo de vida. Especificamente, a atenção se direciona para avaliar a influência existencial dos indivíduos que possuem daqueles que não possuem sentido de vida. Não obstante, essa área buscou explorar os elementos positivos para a saúde mental, em que as pesquisas deram ênfase na identificação dos fatores que auxiliam e elevam a capacidade da pessoa desenvolver e melhorar o bemestar subjetivo na condução de uma vida significativa (Cohen e Cairns, 2012).

A filosofia, psiquiatria e psicologia foram áreas que buscaram o entendimento do conceito de sentido de vida. Assim, contribuíram na especulação sobre os elementos que influenciam o sentido de vida e seu efeito no indivíduo (Cohen e Cairns, 2012). Desse modo, os estudos na literatura indicam que o sentido de vida pode ser alcançando a partir de diferentes formas, tais como: senso de coerência na vida (Battista e Almond, 1973); mediante a necessidade de autoeficácia, propósito de vida e autoestima (Baumeister e Wilson, 1996); objetivos direcionados (Ryff e Singer, 1998). Por sua vez, o lado emocional do sentido de vida abrange o sentimento de felicidade e realização quando as pessoas atendem seus objetivos e as experiências gerais da vida (Cohen e Cairns, 2012).

Apesar dos elementos mais gerais apresentados anteriormente, torna-se fundamental descrever $\mathrm{o}$ impacto que Victor Frankl exerceu na propagação deste construto. Frankl (1989) construiu uma linha de 
abordagem psicoterápica, chamada de Logoterapia e análise existencial, a qual também é chamada de psicoterapia centrada no sentido da vida (Aquino et al., 2015). Esta abordagem, fundamentase na filosofia e apresenta três pressupostos: liberdade de vontade (o ser humano é livre para escolher, mesmo diante dos condicionamentos sociais); vontade de sentido (corresponde a motivação primária da existência humana) e sentido da vida (se existe uma busca de sentido por parte do indivíduo, há também um sentido a ser descoberto nas entrelinhas das situações) (Lukas, 1989).

De acordo com a teoria existencial de Frankl (1989), a razão principal do comportamento humano é a vontade de sentido e assim, o indivíduo se esforça para encontrar um motivo para sua existência (Kleftaras e Psarra, 2012). Neste sentido, o significado procurado ou encontrado pelas pessoas difere entre elas de modo que existe uma dificuldade em estabelecer uma definição geral para esse construto. Apesar disso, mediante as experiências da vida, o ser humano descobre e assume seu próprio dever e se dedica na escolha de um propósito. Uma pessoa pode desvendar o seu significado existencial de vida, mediante aos valores criativos (experiência por meio do alcance da meta e o que contribuiu para vida, a exemplo do trabalho); valores vivenciais (experiências por meio de situações belas ou verdadeiras) e valores atitudinais (tomar uma postura diante de uma situação difícil ou que é inevitavelmente acompanhada pelo sofrimento) (Frankl, 1989).

O sentido de vida é um elemento vital que fornece coerência à percepção de mundo do indivíduo e um aspecto importante para o seu bem-estar psicológico e espiritual. Todavia, Frankl (1989) discute que, quando a pessoa se esforça para encontrar sentido em sua vida, sua saúde psíquica é prejudicada e assim, a falta de sentido causa uma situação chamada de vácuo existencial. Essa sensação de vazio decorre da visão de que a vida não tem sentido e a existência não tem qualquer propósito (Aquino et al., 2015).

Diante dos principais postulados teóricos abarcados por Frankl, o construto sentido de vida tem recebido atenção dos pesquisadores na operacionalização do fenômeno e sua relação com outras variáveis (Damásio, 2013). Neste sentido, surgiram definições similares e diferentes da proposta realizada por Frankl. Reker e Wong (1988) definiram o construto como a apreensão de organização e 
ISSN: 2763-5724 | https://www.periodicojs.com.br/index.php/hs

conexão com a própria existência, associado à busca e a realização de metas/objetivos significativos e que tem como consequência a sensação de felicidade e/ou êxito. Por sua vez, Steger (2009) considera sentido de vida como o grau em que os indivíduos possuem e percebem que têm um propósito ou um objetivo essencial em suas vidas.

Portanto, adota-se na presente dissertação a definição de sentido de vida proposto por Steger (2009), o qual identificou duas dimensões do construto: busca de sentido e presença de sentido. Este autor, igualmente com outros pesquisadores, sistematizaram $\mathrm{o}$ construto e desenvolveram instrumentos para a sua mensuração, sendo apresentados a seguir as principais medidas.

\section{Mensuração}

Inúmeros instrumentos foram desenvolvidos para mensurar sentido de vida, os quais diferem na quantidade de dimensões, número de itens e perspectiva teórica. Nesta direção, esta seção busca apresentar duas medidas que são comumente utilizadas nos estudos acerca deste construto, o Teste de Propósito de Vida (PIL-R) e Questionário de Sentido de Vida (QSV).
$\mathrm{O}$ primeiro instrumento com $\mathrm{o}$ intuito de confirmar as preposições teóricos da Logoterapia e análise existencial foi o Teste de Propósito de Vida (Pil-Teste) proposto por Crumbaugh e Maholick (1964), o qual era composto por três partes (itens, complementação de sentenças e descrição autobiográfica). Devido a sua extensão, Harlow, Newcomb e Bentler (1987) desenvolveram uma versão revisada desta medida, o Pil-R, composto por três dimensões: desespero existencial, realização existencial e vazio existencial, os quais em conjunto, contemplam os elementos de propósito na vida, liberdade, medo da morte, satisfação com a própria vida, se a vida vale a pena e ideias suicidas (Aquino et al., 2015). Esta medida foi adaptada para o contexto brasileiro por Aquino et al. (2009), os quais encontraram uma estrutura bifatorial, correspondendo as dimensões realização existencial e vazio existencial.

Por sua vez, o Questionário Sentido de Vida foi desenvolvido por Steger et al. (2006) para avaliar as dimensões presença e busca de sentido, sendo composta por dez itens distribuídos igualmente entre os subfatores, respondidos em uma escala de sete pontos. A presença de sentido se 
ISSN: 2763-5724 | https://www.periodicojs.com.br/index.php/hs

refere àquelas pessoas que estão empenhadas em um significado ao longo da vida, enquanto busca do sentido diz respeito àquelas que estão tentando compreender ou elevar o propósito em sua vida. No Brasil, sua estrutura bidimensional foi confirmada por Aquino et al. (2015) e Damásio e Koller (2015).

Correlatos de sentido de vida com o bem-estar

Pesquisas demonstram o impacto negativo provocado pelo vazio existencial no indivíduo, tais como estresse percebido (Bauer-Wu e Farran, 2005), sintomas de ansiedade e depressão (Riichiro e Masahiko, 2006) e ideação suicida (Edwards e Holden, 2001). Por outro lado, a presença de sentido expressa impacto positivo no bem-estar psicológico (Ho, Cheung e Cheung, 2010), saúde (Brassai, Piko, e Steger, 2011) e prevenção da depressão e ansiedade em pacientes com câncer (Vehling et al., 2011). Assim, observamse as evidências da importância desse construto no bom funcionamento psicológico.

Nesta perspectiva, Kleftaras e Psarra (2012) realizaram uma pesquisa na Grécia com o objetivo de verificar as relações entre as dimensões sentido de vida, sintomatologia depressiva e saúde psicológica geral. Para isso, os autores contaram com a participação de 401 jovens recentemente convocados para o serviço nacional da marinha. Os resultados demonstraram que os indivíduos com alto escore no questionário de depressão apresentaram baixa pontuação na escala sentido de vida. Além disso, encontraram que as pessoas com alto sentido de vida demonstraram maior saúde psicológica, bem como também maior significado existencial; isso também ocorreu para pessoas casadas e maiores participações em atividades sociais. Desse modo, indivíduos que experimentam maior sentido de vida, possuem uma percepção positiva da saúde e menor índice de sintomas negativos (e.g., ansiedade, distúrbios de sono) (Kleftaras e Psarra, 2012).

Cohen e Cairns (2012) buscaram encontrar quais construtos positivos (e.g., felicidade, autoeficácia) moderam a relação entre busca de sentido e bemestar subjetivo. Para tal, participaram 500 pessoas da população geral da Austrália. A partir das análises, os dados apresentaram a relação negativa entre altos níveis de busca de sentido e bemestar subjetivo, além dos efeitos moderados que a de presença de sentido 
ISSN: 2763-5724 | https://www.periodicojs.com.br/index.php/hs

e autorrealização possuem na felicidade quando os indivíduos buscam sentido. Os autores concluem que a presença de sentido na vida e alto nível de realização, podem auxiliar o sofrimento quando a pessoa busca por significado existencial. Damásio e Koller (2015), na mesma direção do estudo anterior, encontraram no contexto brasileiro que busca de sentido modera a relação entre as dimensões de significado existencial e bem-estar subjetivo.

Por sua vez, Aquino et al. (2009) realizou um estudo no Brasil para verificar a relação entre sentido de vida e atitude religiosa. Com a participação de 300 pessoas da população-geral, os resultados indicaram uma correlação positiva entre atitude religiosa e realização existencial. Tais autores concluíram que a crença em algo superior, pode contribuir na relação de sentido do indivíduo no mundo. Por sua vez, Aquino, Dará e Simeão (2016) objetivaram investigar a relação entre depressão, sentido de vida e percepção ontológica de tempo em 110 pacientes que estavam em psicoterapia. Os resultados evidenciaram correlações negativas entre depressão, sentido de vida e percepção do passado, presente e futuro, evidenciando que presença de sentido e as dimensões ontológicas do tempo contribuem como construtos

protetores na expressão da sintomatologia depressiva.

Diante do que foi exposto, observa-se como o construto sentido de vida é importante para o funcionamento psíquico saudável. Os estudos demonstram que a presença de sentido fornece significado existencial ao indivíduo e consequentemente, maior bem-estar psicológico. Não obstante, sentido de vida é um dos diferentes construtos na promoção de qualidade psíquica das pessoas, podendo-se citar outros fenômenos, a exemplo da autoestima. Desse modo, o próximo tópico irá detalhar este fenômeno.

\subsection{Autoestima}

O desenvolvimento da autoestima

A ciência comportamental, desde o seu início, expressa interesse no desenvolvimento da autoestima, a qual seus teóricos sugerem que o modo como os indivíduos se sentem em relação a si decorre em grande parte de como os outros pensam e sentem a seu respeito (MacDonald e Leary, 2012). A perspectiva interacionista simbólica defendida por Cooley (1902) e Mead (1934) definiu a autopercepção e autoestima como a percepção que os indivíduos tem acerca de como as outras 
pessoas a percebem. Desse modo, diferentes pesquisadores estudaram as origens interpessoais da autoestima (MacDonald e Leary, 2012).

A perspectiva dos interacionistas simbólicos no desenvolvimento da autoestima oferece a ideia que as opiniões dos outros influenciam no período da infância e assim, os parentes/cuidadores que as aprovam, nutrem e reagem tendem a propiciar na criança uma autoestima mais elevada quando comparado aos parentes que desaprovam e não se interessam por elas (Coopersmith, 1967; Harter, 1999; Ojanen e Perry, 2007). Apesar da relação entre os pais-filhos influenciarem na autoestima, na adolescência, a aprovação entre os amigos e colegas se torna forte preditor da autoestima (Harter, Whitesell e Junkin, 1998), além dos relacionamentos com pessoas importantes durante o ciclo de vida (e.g., avós, professores) que fornecem apoio e orientação (MacDonald e Leary, 2012). Tais achados corroboram a ideia do impacto da percepção dos outros no desenvolvimento da autoestima.

Ainda sobre o desenvolvimento da autoestima, recentemente, Kuster e Orth (2013) investigaram se a estabilidade individual deste fenômeno pode declinar em decorrência do tempo.
Para tal objetivo, realizaram seis avaliações em um período de 29 anos (N = 3.180; idade variando de 14 a 102 anos); os resultados demonstraram que, à medida que os intervalores teste-reteste aumentaram, os coeficientes de estabilidade declinaram exponencialmente e assintoticamente, aproximaram-se de um valor diferente de zero (estimado em 0,13 na amostra total). Diante dos resultados, os autores discutiram que a natureza do construto corresponde à um traço de personalidade, uma vez que a estabilidade da autoestima no período de tempo avaliado foi similar a estabilidade das dimensões dos cinco grandes traços de personalidade (Hampson e Goldberg, 2006).

Desse modo, dada a corroboração dos estudos sobre a consideração da autoestima como traço, MacDonald e Leary (2012), por outro lado, argumentam que os fatores no desenvolvimento da característica individual da autoestima não abarcam totalmente a aprovação e aceitação explícitas dos pares. Nessa direção, a autoestima elevada também se associa à crença de que uma pessoa possui atributos positivos (e.g., aparência atraente, habilidades artísticas, música, esportes). $\mathrm{Na}$ verdade, a pessoa que 
acredita nos seus atributos desejáveis, confiam que essas características promovem aceitação social (MacDonald, Saltzman, e Leary, 2003), evidenciando assim a importância que a percepção dos outros exerce na autoestima.

Diante disso, de acordo com a revisão realizada por Orth e Robins (2014), três pontos são considerados na caracterização da autoestima. Primeiro, apesar dos indivíduos diferirem de forma particular durante a trajetória de vida, os estudos longitudinais sugerem que a autoestima tende a aumentar entre a adolescência e a metade da idade adulta, sendo o auge entre 50-60 anos, e diminui rapidamente após essa faixa etária (Orth, Robins, e Widaman, 2012). Segundo esses autores, a autoestima é relativamente estável, porém não é um traço imutável, a qual o nível de estabilidade pode ser comparado com as características básicas da personalidade, como extroversão e conscienciosidade (Hampson e Goldberg, 2006). Por último, observaram que a autoestima é um importante preditor de variáveis durante a vida (e.g., saúde, relacionamentos, trabalho; Baumiester, Campbell, Krueger, e Vohs, 2003). Considerando que a autoestima é um fenômeno importante na manutenção de outros construtos de componente afetivo e comportamental, pesquisadores começaram a despender esforços para mensurar esse construto, a exemplo de Rosenberg (1969) e Coopersmith (1967).

\subsubsection{Mensuração}

A autoestima é um importante construto na área psicológica. Neste sentido, diferentes pesquisadores se dedicaram em sua mensuração para investigar seus antecedentes e consequentes. Entre as diversas escalas existentes, tais como Janis and Field Feelings of Inadequacy (Janis e Field, 1959), Tennesse Self-Concept (Roid e Fitss, 1988) e Ziller Social Self-Esteem Scale (Ziller, Hagey, Smith, e, Long, 1969) observam-se que as medidas mais utilizadas na literatura são a Scale SelfEsteem (Ronseberg, 1965) e Self-Esteem Inventory (Coopersmith, 1967), sendo as mesmas detalhadas a seguir.

A Escala de Autoestima de Rosenberg foi originalmente desenvolvida por Rosenberg (1965) para mensurar de forma global os sentimentos da autoestima e autoaceitação. A escala é composta por dez itens, os quais compõe a unidimensionalidade da autoestima. Os respondentes reportam diretamente quais são seus sentimentos em relação a si, com a escala de resposta 
que varia entre 1 (Discordo fortemente)

e 4 (Concordo fortemente). Estudos divergem quanto a estrutura da escala, pois pesquisadores encontram a estrutura original, enquanto outros identificam uma estrutura bidimensional (Blascovich e Tomaka, 1994). No contexto brasileiro, observa-se que essa é a medida mais utilizada nos estudos acerca da autoestima, a exemplo dos estudos de Hutz e Zanon (2011), os quais encontram uma estrutura unidimensional, enquanto Sbicigo, Bandeira e Dell'Aglio (2010), na população adolescente, identificaram uma estrutura bidimensional.

O Inventário de Autoestima desenvolvido por Coopersmith (1967), por sua vez, avalia a evolução de atitudes sobre diferentes domínios do self. No primeiro momento, a medida foi composta por 50 itens, os quais mensuravam a autoestima na população infantil e consistia em quatro fatores: eu geral, grupo social, família e escola. Em outro trabalho subsequente, Coopersmith (1975) criou o inventário de autoestima (forma B), no qual selecionou 25 itens da medida original e abarcava a unidimensionalidade da autoestima. As escalas de resposta de ambas as medidas são dicotômicas, sendo expressa a partir de descritores como "tem a ver comigo" ou "não tem a ver comigo" (Blascovich e Tomaka, 1994). No Brasil, foram encontrados os estudos realizados por Gobitta e Guzzo (2002), utilizando a forma- $A$, enquanto Santos (2008) utilizou em seu estudo a forma-B; entretanto, não foram identificados estudos que versassem sobre as propriedades psicométricas do inventário de coopersmith.

Correlatos da autoestima com o bemestar

Como já explanado no tópico anterior, a autoestima se refere a percepção geral que o indivíduo possui do seu valor e estima (Rosenberg, 1979). Neste sentido, identifica-se uma miríade de construtos que são explicados pela autoestima, a exemplo de características demográficas e relacionamentos sociais (Bosson, Swann, e Pennebaker, 2000; Diener e Diener, 1995). Ademais, a autoestima vem demonstrando forte correlação com os componentes do bemestar subjetivo em diferentes estudos, os quais indivíduos com níveis elevados de autoestima apresentam alta satisfação com a vida e afetos positivos e baixo nível de afetos negativos (Bosson et al., 2000; Kong, Zhao, e You, 2013).

Nesta perspectiva, Smedema, Catalano e Ebener (2010) buscaram examinar a relação entre coping e bemestar subjetivo mediante a mediação da 
autoestima. Para tal, participaram do estudo 242 pessoas que constavam lesões na medula espinhal $\left(\mathrm{M}_{\text {idade }}=44,6\right.$ anos; $\mathrm{DP}=13,2)$. Os resultados demonstraram correlações positivas entre bem-estar subjetivo, autoestima e as dimensões coping positiva (esperança, proativo e senso de humor). Dessa forma, os autores desenvolveram um modelo explicativo, em que coping positivo e coping negativo se influenciam mutuamente na predição da autoestima e bem-estar subjetivo, embora diferem entre si na direção de predição da autoestima e bem-estar subjetivo. Assim, concluíram que estratégias positivas de resolução de problema, eleva o nível de autoestima e consequentemente o bem-estar subjetivo, propiciando efeito positivo na saúde mental (Smedema et al., 2010).

Kong et al. (2013) objetivaram examinar os efeitos de mediação e moderação da autoestima global na relação entre suporte social e bem-estar subjetivo na população chinesa. Participaram $391 \quad$ estudantes universitários com média de idade de 20,3 anos (DP = 1,84). As análises de correlação viabilizaram a análise de mediação, a qual foi consistente no modelo proposto de suporte social $\rightarrow$ autoestima $\rightarrow$ bem-estar subjetivo (satisfação com a vida, afetos positivos e afetos negativos). Por sua vez, ao verificar o papel moderador da autoestima entre suporte social e bemestar subjetivo, especificamente verificou-se que a moderação ocorreu na relação entre suporte social e satisfação com a vida e afetos positivos, não o fazendo com os afetos negativos.

Diante dos resultados, os autores concluem que indivíduos com altos níveis de apoio social, tendem apresentar maior autoestima global e consequentemente, contribui nas avaliações de sua vida e afetos positivos; além disso, pessoas com alto nível de autoestima e suporte social relataram maiores pontuações em satisfação com a vida e afetos positivos, comparados com aqueles com baixo suporte social (Kong et al., 2013). No que se refere ao contexto brasileiro, Hutz et al. (2014) encontraram relações positivas entre autoestima e bem-estar subjetivo, corroborando os estudos descritos anteriormente. Portanto, apresentado as evidências de sentido de vida e autoestima no bem-estar subjetivo, fazse necessário descrever a contribuição de outro importante construto na saúde psíquica das pessoas: otimismo.

\subsection{Otimismo}


Definição

A construção do conceito de otimismo possui várias facetas (Kleiman et al., 2015), embora na literatura possuam um maior destaque para duas concepções teóricas, o Otimismo explicativo e o Otimismo disposicional. O otimismo explicativo, também conhecido como explanotory style, busca compreender como as pessoas explicam a ocorrência de situações boas ou ruins que sucedem na sua rotina de modo que não se limita apenas a um pensamento positivo (Peterson e Steen, 2009). Neste sentido, um indivíduo que possui um estilo explicativo pessimista, por exemplo, atribui aos eventos ruins que acontecem em sua vida a causas internas, estáveis e comuns; enquanto atribui a ocorrência de uma situação favorável a causas externas, instáveis e incomuns. Entretanto, a pessoa que adota um estilo explicativo otimista acredita que as causas dos eventos satisfatórios são internos, estáveis e comuns, atribuindo de modo contrário para situações adversas (Seligman, 1998).

Por sua vez, o otimismo disposicional, refere-se a uma expectativa generalizada para as ocorrências de eventos futuros de modo que há pessoas com expectativas positivas (otimistas), enquanto existem indivíduos com expectativas negativas (pessimistas) diante de algum acontecimento (Carver e Scheier, 2009). Dessa forma, as pessoas otimistas e pessimistas lidam de maneira diferente com as adversidades das situações. Por outro lado, o ponto comum entre os otimistas e pessimistas possuem são as expectativas frente ao futuro, embora sejam em direções opostas, pois o otimista apresenta uma expectativa positiva em relação ao futuro, quanto o pessimista possui uma expectativa negativa (Scheier e Carver, 1993). Considerando isso, seus proponentes teorizaram o construto mediante ao modelo motivacional expectancy-value.

Especificamente, o modelo motivacional expectancy-value possui como pressuposto de que o comportamento se organiza por meio da busca de objetivos/metas. Apesar do conceito de metas variarem entre os teóricos, Carver e Scheier (2009) as definem como estados ou ações que as pessoas percebem como desejáveis ou indesejáveis. Os indivíduos se esforçam para regular seus comportamentos ao que consideram desejável e tentam evitar aquilo que entendem como indesejável. Desse modo, quanto mais importante for o objetivo para a pessoa, maior será o seu valor dentro da motivação. Por sua vez, 
a expectativa diz respeito a um sentimento de confiança ou dúvida sobre a capacidade de obtenção do valor da meta. Indivíduos que possuem maior confiança, tendem a agir mais e o esforço persiste mesmo diante de eventuais adversidades (Carver e Scheier, 2014).

Diante disso, otimismo e pessimismo variam em um contínuo, constituindo uma dimensão de personalidade. Carver e Scheier (2014) para sustentar esse argumento, exemplificam que a diferença entre o traço otimista e os big five se concentra na formação de domínios de conteúdo. À respeito disso, os autores discutem que os cinco grandes traços representam "o quê" (por exemplo, extroversão como comunicação social), enquanto a dimensão do otimismo parte do "como" das motivações humanas (como os objetivos se transformam em comportamento).

Ademais, o otimismo é um construto cognitivo dado às expectativas aos resultados futuros, de forma que se associa com à motivação na medida em que indivíduos otimistas exercem esforços para realizar seus objetivos (Scheier, Carver, e Segerstrom, 2010). Neste sentido, compreende-se a natureza do construto otimismo como disposicional, uma vez que compõe uma característica intrínseca da pessoa, bem como abarca o elemento motivacional. Em outras palavras, o otimismo é um fenômeno que interliga os processos cognitivo, emocional e motivacional, pois ao mesmo tempo há a expectativa, $o$ sentimento em relação ao objeto e as implicações motivacionais para realizar o que o indivíduo almeja (Carver e Scheier, 2014).

\section{Mensuração}

Entre as medidas existentes na psicologia para a mensuração do otimismo, a que se apresenta com maior destaque no campo da literatura diz respeito a Life Orientation Test (LOT) desenvolvida por Scheier e Carver (1985). Todavia, este instrumento sofreu diferentes críticas acerca da similaridade com outros construtos, a exemplo da autoestima (Bastianello, Pacico, e Hutz, 2014). Neste sentido, Scheier, Carver e Bridges (1994) revisaram o instrumento e propuseram uma nova medida, a Revised Life Orientation Test (LOT-R), no qual os itens buscam avaliar expectativas positivas e negativas.

A LOT-R é um instrumento unidimensional composto por dez itens, os quais seis avaliam a natureza do construto e quatro são itens distratores (Bastianello et al., 2014). Sua estrutura fatorial explicou 48,1\% da variância, 
apresentando consistência interna satisfatória $(\alpha=0,78)$. No contexto brasileiro, essa medida foi adaptada por Bastianello et al. (2014) com estudantes universitários, os quais encontraram uma estrutura unidimensional e alfa de Cronbach de 0,80 . Por outro lado, Ottati e Noronha (2017) aplicaram esse instrumento para adolescentes, as quais verificaram uma estrutura bifatorial, isto é, as dimensões otimismo e pessimismo.

A principal crítica em relação a LOT-R, refere-se a inconsistência da sua estrutura fatorial (Ottati e Noronha, 2017), embora Carver e Scheier (2014) defendem a medida como unidimensional. Nesta perspectiva, Pedrosa et al. (2015) desenvolveram uma nova medida na avaliação do otimismo disposicional na tentativa de contrapor as limitações apresentadas pela LOT-R. Desse modo, os autores elaboraram dez itens compondo The Optimism Scale. Esta medida foi respondida por 2.693 participantes da Espanha, encontrando uma estrutura fatorial unidimensional, com $51,1 \%$ de variância explicada e consistência interna satisfatória $(\alpha=$ 0,84). Esta medida apresenta evidências de validade fatorial e confiabilidade no contexto brasileiro e inglês, os quais demonstraram indicadores psicométricos adequados (Coelho et al., 2018).

Correlatos do otimismo com o bemestar

O bem-estar é um componente essencial na saúde mental de modo que reflete no ajustamento favorável na qualidade de vida dos indivíduos (Mishra, 2013). Schweizer, Beck-Setffer e Schneider (1999) observaram que o otimismo influencia na percepção individual do bem-estar dada a relação próxima entre as expectativas sobre o futuro das pessoas. Neste sentido, o otimismo é positivamente correlacionado com satisfação de vida, saúde mental e autoestima (Strassle, Mckee, e Plant, 1999), com afetos positivos (Avia, 1997) e bom humor (Peterson, 2000).

Os indivíduos otimistas em relação aos pessimistas apresentam melhor saúde física (Burke, Joyner, Czech, e Wilson, 2000). Peterson (2000) demonstrou que em termos de saúde física, otimismo é um atributo de personalidade que modera níveis de estresse. Scheier e Carver (1987), por sua vez, propuseram que a influência entre otimismo e saúde física e bem-estar pode ser devido ao uso de estratégias de enfrentamento mais eficazes por indivíduos otimistas ao lidar com o 
ISSN: 2763-5724 | https://www.periodicojs.com.br/index.php/hs

estresse. Além disso, pessoas otimistas ao persistirem nos seus objetivos desejados apresentam maiores indicadores de bem-estar, assim como, crenças adaptativas e persistência na busca de metas (Scheier et al., 2010).

Hanssen et al. (2015) buscaram investigar como a estratégia de enfrentamento motivacional e o otimismo se relacionam como indicadores do bem-estar (qualidade de vida, depressão, ansiedade e enfermidade física). Para isso, contaram com a participação de 254 indivíduos holandeses $\left(\mathrm{M}_{\mathrm{idade}}=35,31\right.$ anos; $69 \%$ sexo feminino). Os resultados demonstraram que o otimismo se relaciona com os indicadores do bemestar mediado pelas dimensões flexibilidade das metas e persistência dos objetivos. Os autores concluem que esses resultados contribuem para a ideia de que a flexibilidade das metas protege as pessoas de consequências negativas em circunstâncias estressantes e auxilia na promoção da qualidade de vida. Portanto, a partir dos estudos supracitados pode-se observar as evidências empíricas da relação do otimismo e bem-estar psicológico.

\section{Conclusions}

apresentar os modelos teóricos em torno do bem-estar. Além disso, a relação entre os principais construtos ao longo do capítulo (sentido de vida, autoestima e otimismo), uma vez que seus conceitos, mensuração e correlação com o bemestar foram apresentados de forma separada. Como pode ter sido observado, o ponto comum entre eles é o impacto na qualidade de vida das pessoas, sobretudo, na saúde mental. Assim, o conhecimento de estudos que versam sobre as relações entre si se torna importante.

Em relação ao sentido de vida e autoestima, Zhang et al. (2015) buscaram investigar como o sentido de vida e autoestima podem impactar no vício de internet, dado que esta variável tem sido reconhecida como mal adaptativa para o funcionamento psíquico. Os resultados demonstram que o sentido de vida e autoestima oferecem proteção contra o vício de internet. Os autores concluem a importância de trabalhar em conjunto os dois construtos como um fator protetivo a desajustamentos psicológicos.

Por sua vez, relacionando significado existencial e otimismo, Ju et al. (2013) objetivaram verificar os efeitos de sentido de vida e otimismo no 
ISSN: 2763-5724 | https://www.periodicojs.com.br/index.php/hs

bem-estar em uma comunidade de população idosa. Os pesquisadores elaboraram a hipótese que o otimismo prediz o bem-estar subjetivo mediado pelo sentido de vida. Por meio de uma equação estrutural, os autores corroboraram sua hipótese e evidenciaram que o significado de vida e o otimismo reflete um funcionamento psicológico positivo, contrapondo a aflição e angústia presente em geral neste estágio da vida.

Por sua vez, acerca da relação entre autoestima e otimismo, Mäkikangas e Kinnunen (2003) realizaram um estudo longitudinal (dois tempo, intervalo de um ano), para verificar como a autoestima e otimismo influenciam no estresse psicossocial e bem-estar em trabalhadores finlandeses. Os resultados demonstraram que baixos níveis de autoestima e otimismo tiveram um efeito negativo no desgaste emocional e sofrimento psíquico no sexo masculino; por outro lado, no sexo feminino o baixo nível de otimismo moderou a relação entre clima organizacional ruim, esgotamento emocional e o desgaste mental. As autoras concluíram a importância dessas variáveis como fatores protetivos em contextos mais específicos, como o trabalho.
Portanto, esses estudos evidenciam como os construtos sentido de vida, autoestima e otimismo se relacionam com o bem-estar. É importante ressaltar, diante do que foi demonstrado, que essas variáveis são conceituadas e medidas de diferentes formas, porém, apresentam como elemento em comum a promoção da qualidade de vida dos indivíduos. Assim, na presente pesquisa deste trabalho, considera esses três construtos como bem-estar subjetivo, uma vez que este é um fenômeno complexo e multidimensional e não se reduz a um único modelo ou definição, mas sim, na pluralidade de variáveis que contribuem para o ajustamento psicológico saudável.

\section{REFERENCES}

Aquino, T. A. A. D. et al. (2009). Atitude religiosa e sentido da vida: um estudo correlacional. Psicologia: Ciência e Profissão, 29, 228-243.

Aquino, T. A. A. D., Dará, D. M. B., Simeão, S. D. S. S. (2016). Depressão, percepção ontológica do tempo e sentido da vida. Revista Brasileira de Terapias Cognitivas, 12, 35-41.

Aquino, T. A. A. et al. (2015). Questionário de sentido de vida: evidências de sua validade fatorial e 
ISSN: 2763-5724 | https://www.periodicojs.com.br/index.php/hs

consistência interna. Psicologia: Ciência e Profissão, 35, 4-19.

Avia, M. D. (1997). Personality and positive outcomes. European Journal of Personality, 11, 33-56.

Bastianello, M. R., Pacico, J. C., Hutz, C. S. (2014). Optimism, self-esteem and personality: adaptation and validation of the Brazilian Version Of The Revised Life Orientation Test (LOT-R). PsicoUSF, 19, 523-531.

Battista, J., Almond, R. (1973). The development of meaning in life. Psychiatry, 36, 409-427.

Bauer-Wu, S., Farran, C. J. (2005). Meaning in life and psycho-spiritual functioning: a comparison of breast cancer survivors and healthy women. Journal of Holistic Nursing, 23, 172-190.

Baumeister, R. F.,Wilson, B. (1996). Life stories and the four need for meaning. Psychological Inquiry, 7, 322325.

Baumeister, R. F. et al. (2003). Does high self-esteem cause better performance, interpersonal success, happiness, or healthier lifestyles? Psychological Science in the Public Interest, 4, 1-44.

Blascovich, J., Tomaka, J. (1994). Measures of self-esteem. In J. P. Robinson, P. R. Shaver, e L. S.
Wrightsman (Eds.), Measures of personality and social psychological atitudes. San Diego: Academic Press. (115-160).

Bosson, J. K., Swann, W. B., Pennebaker, J. W. (2000). Stalking the perfect measure of implicit selfesteem: The blind men and the elephant revisited? Journal of Personality and Social Psychology, 79, 631-643.

Brassai, L., Piko, B. F., Steger, M. F. (2011). Meaning in life: Is it a protective factor for adolescents' psychological health?. International Journal of Behavioral Medicine, 18, 44-51.

Burke, K. L., Joyner, A. B., Czech, D. R., Wilson, M. J. (2000). An investigation of concurrent validity between two optimism/pessimism questionnaires: The life orientation test-revised and the optimism/pessimism scale. Current Psychology: Development, Learning, Personality, Social, 19, 129-136.

Carver, C. S., M. F. Scheier (2009). Optimism. In C. R. Snyder e S. L. Lopez. $\left(2^{\circ}\right.$ Eds.) Handbook of positive psychology. New York: Oxford University Press.

Carver, C. S., Scheier, M. F. (2014). Dispositional optimism. Trends in Cognitive Sciences, 18, 293-299.

Cohen, K., Cairns, D. (2012). Is searching for meaning in life associated 
ISSN: 2763-5724 | https://www.periodicojs.com.br/index.php/hs

with reduced subjective well-being?

Confirmation

and

possible

moderators. Journal of

Happiness

Studies, 13, 313-331.

Cooley, C. H. (1902). Human nature and the social order. New York: Scribner's.

Coopersmith, S. (1967). The antecedents of self-esteem. San Francisco,

CA: Freeman.

Crumbaugh, J. C., Maholick, L. T. (1964). An experimental study in existentialism: The psychometric approach to Frankl's concept of noogenic neurosis. Journal of Clinical Psychology, 20, 200-207.

Damásio, B. F. (2013). Sentido de vida e bem-estar subjetivo: interações com esperança, otimismo, autoeficácia e autoestima em diferentes etapas do ciclo vital. Tese de Doutorado. Universidade Federal do Rio Grande do Sul, Rio Grande do Sul, RS, Brasil.

Damásio, B. F., Koller, S. H. (2015). How search for meaning interacts with complex categories of meaning in life and subjective well-being?. The Spanish Journal of Psychology, 18, 1-10.

Diener, E. (1984). Subjective wellbeing. Psychological Bulletin, 95, 542575.

Diener, E. (2013). The remarkable changes in the science of subjective well- being. Perspectives on Psychological

Science, 8, 663-666.

Diener, E., Diener, M. (1995). Crosscultural correlates of life satisfaction and self-esteem. Journal of Personality and Social Psychology, 68, 653-663.

Diener, E. et al. (2017). Findings all psychologists should know from the new science on subjective wellbeing. Canadian

Psychology/Psychologie canadienne, 58, 87-184.

Diener, E., Oishi, S., Lucas, R. E. (2003). Personality, culture, and subjective wellbeing: Emotional and cognitive evaluations of life. Annual Review of Psychology, 54, 403-425.

Diener, E. et al. (2017). If, why, and when subjective well- being infl uences health, and future needed research. Applied Psychology: Health and WellBeing, 9,133-167.

Edwards, M. J., Holden, R. R. (2001). Coping, meaning in life, and suicidal manifestations: Examining gender differences. Journal of Clinical Psychology, 57, 1517-1534.

Eid, M., Larsen, R. J. (Eds.). (2008). The science of subjective well- being. New York, NY: Guilford Press.

Fava, G. A. (2016). Well-being therapy: current indications and emerging 
perspectives. Psychotherapy

and

Psychosomatics, 85, 136-145.

Fitts, W. H., Roid, G. H. (1964). Tennessee self concept scale. Nashville, TN: Counselor Recordings and Tests.

Frankl, V. E. (1989). Psicoterapia e sentido da vida (A. M. Castro, trad.). São Paulo: Quadrante. (Trabalho original publicado em 1946).

Giacomoni, C.H. (2004). Bem-estar subjetivo: em busca da qualidade de vida. Temas em Psicologia, 12, 43-50.

Gobitta, M., Guzzo, R. S. L. (2002). Initial study of the Self-Esteem Inventory (SEI): Form A. Psicologia: Reflexão e Crítica, 15, 143-150.

Guerra, V. M. et al. (2017). Psicologia positiva: seus construtos e a sua relação com a psicologia social. In C. F. Lima e C. E. Pimentel (Org.). Revisitando a psicologia social. Curitiba: Juruá (127148).

Hampson, S. E., Goldberg, L. R. (2006). A first large cohort study of personality trait stability over the 40 years between elementary school and midlife. Journal of Personality and Social Psychology, 91, 763-779.

Hanssen, M. M. et al. (2015). Optimism, motivational coping and well-being: Evidence supporting the importance of flexible goal adjustment. Journal of Happiness Studies, 16, 1525-1537.

Harlow, L. L., Newcomb, M. D., Bentler, P. M. (1987). Purpose in Life Test assessment using latent variable methods. British Journal of Clinical Psychology, 26, 235-236.

Harter, S. (1999). The construction of the self: A developmental perspective. New York: Guilford Press.

Harter, S., Whitesell, N. R., Junkin, L. J. (1998). Similarities and differences in domain specific and global selfevaluations of learning disabled, behaviorally disordered, and normally achieving adolescents. American Education Research Journal, 35, 653680 .

Henriques, G. (2011). A new unified theory of psychology. New York, NY: Springer.

Henriques, G., Kleinman, K., Asselin, C. (2014). The Nested Model of well-being: A unified approach. Review of General Psychology, 18, 7-18.

Ho, M. Y., Cheung, F. M., Cheung, S. F. (2010). The role of meaning in life and optimism in promoting wellbeing. Personality and Individual Differences, 48, 658-663.

Hutz, C. S., Zanon, C. (2011). Revisão da adaptação, validação e normatização da escala de autoestima de 
ISSN: 2763-5724 | https://www.periodicojs.com.br/index.php/hs

Rosenberg. Avaliação Psicológica, 10, 41-49.

Hutz, C. S., et al. (2014). The relationship of hope, optimism, selfesteem, subjective well-being, and personality in brazilians and americans. Psychology, 5, 514-522.

Ishida, R., Okada, M. (2006). Effects of a firm purpose in life on anxiety and sympathetic nervous activity caused by emotional stress: assessment by psychophysiological method. Stress and health: Journal of the International Society for the Investigation of Stress, 22, 275-281. Janis, I. L., Field, P. B. (1959). Sex differences and personality factors related to persuasibility. In C. I. Hovland e I. L. Janis (Eds.), Personality and persuasibility (pp. 55-68). Oxford, England: Yale Univer. Press.

Ju, H. et al. (2013). Mediational effect of meaning in life on the relationship between optimism and well-being in community elderly. Archives of Gerontology and Geriatrics, 56, 309313.

Kleftaras, G., Psarra, E. (2012). Meaning in life, psychological well-being and depressive symptomatology: A comparative study. Psychology, 3, 337345.

Kleiman, E. M. et al. (2017). Optimism and well-being: a prospective multi- method and multi-dimensional examination of optimism as a resilience factor following the occurrence of stressful life events. Cognition and Emotion, 31, 269-283.

Kong, F., Zhao, J., You, X. (2013). Selfesteem as mediator and moderator of the relationship between social support and subjective well-being among Chinese university students. Social Indicators Research, 112, 151-161.

Kuster, F., Orth, U. (2013). The longterm stability of self-esteem: Its timedependent decay and nonzero asymptote. Personality and Social Psychology Bulletin, 39, 677-690.

Lukas, E. (1989). Logoterapia: “A força desafiadora do espírito" (J. de Sá Porto, trad.). São Paulo: Edições Loyola e Leopoldianum.

Lyubomirsky, S., King, L., Diener, E. (2005). The benefi ts of frequent positive affect:Does happiness lead to success? Psychological Bulletin, 31, 803-855.

MacDonald, G. Leary, M. R. (2012). Individual differences in self-esteem. In: M. R., Leary e J. P. Tangney (Eds.). Handbook of self and identity. New York, NY: Guilford Press (pp. 354377).

MacDonald, G., Saltzman, J. L., Leary, M. R. (2003). Social approval and trait 
ISSN: 2763-5724 | https://www.periodicojs.com.br/index.php/hs

self-esteem. Journal of Research in Personality, 37, 23-40.

Maddux, J. (2018). Subjective wellbeing and life satisfaction: an introducion to conceptions, theories, and measures. In: J. Maddux (Ed.). Subjective well-being and life satisfaction. New York: Routledge (pp.3-32).

Mead, G. H. (1934). Mind, self, and society. Chicago: University of Chicago Press.

Miller Smedema, S., Catalano, D., Ebener, D. J. (2010). The relationship of coping, self-worth, and subjective wellbeing: A structural equation model. Rehabilitation Counseling Bulletin, 53, 131-142.

Mishra, K. K. (2013). Optimism and well-being. Social Science International, 29, 75-87.

Ojanen, T., Perry, D. G. (2007). Relational schemas and the developing self: Perceptions of mother and of self as joint predictors of early adolescents' self-esteem. Developmental Psychology, 43,1474-1483.

Orth, U., Robins, R. W. (2014). The development of self-esteem. Current Directions in Psychological Science, 23, 381-387.

Ottati, F., Noronha, A. P. P. (2017). Factor structure of the Life Orientation
Test-Revised (LOT-R). Acta Colombiana de Psicología, 20, 32-39.

Pedrosa, I. et al. (2015). Cuestionario para la evaluación del optimismo: Fiabilidad y evidencias de validez. Terapia Psicológica, 33, 127138.

Peterson, C. (2000). The future of optimism. American Psychologists, 55, 44-55.

Peterson, C., Steen, T. A. (2009). Optimism. In C. R. Snyder e S. L. Lopez. $\left(2^{\circ}\right.$ Eds.) Handbook of positive psychology. New York: Oxford University Press.

Reker, G. T., Wong, P. T. (1988). Aging as an individual process: Toward a theory of personal meaning. In J. E. Birren e V. L. Bengston (Eds.), Emergent theories of aging. New York: Springer (pp. 214-246).

Rosenberg, M. (1979). Conceiving the self. New York: Basic Books.

Ruini, C., Ryff, C. D. (2016). Using eudaimonic well-being to improve lives. In A. M. Wood e J. Johnson (Eds.), The Wiley handbook of positive clinical psychology. New York: Wiley (153166).

Ryan, R. M., Deci, E. L. (2001). On happiness and human potentials: A review of research on hedonic and 
eudaimonic well-being. Annual Review of Psychology, 52, 141-166.

Ryan, R. M., Deci, E. L. (2001). On happiness and human potentials: A review of research on hedonic and eudaimonic well-being. Annual Review of Psychology, 52, 141-166.

Ryff, C. D. (1989). Happiness is everything, or is it? Explorations on the meaning of psychological wellbeing. Journal of Personality and Social Psychology, 57, 1069-1081.

Ryff, C. D. (2014). Psychological wellbeing revisited: Advances in the science and practice of eudaimonia. Psychotherapy and Psychosomatics, 83, 10-28.

Ryff, C. D. (2016). Beautiful ideas and the scientificeEnterprise: sources of intellectual vitality in research on eudaimonic well-being. J. Vitters $\emptyset$ (Ed.). Handbook of eudaimonic well-being. Cham: Springer International Publishing (95-108).

Ryff, C. D., Singer, B. (1998). The contours of positive human health. Psychological Inquiry, 9, 1-28.

Santos, A. M. L. D. (2008). Autoestima e suporte social em mães adolescentes: Um estudo no estado do Amapá-Brasil. Dissertação de Mestrado. Instituto Superior de Psicologia Aplicada, Lisboa, Portugal.
Sbicigo, J. B., Bandeira, D. R., Dell'Aglio, D. D. (2010). Escala de Autoestima de Rosenberg (EAR): validade fatorial e consistência interna. Psico-USF, 15, 395-403.

Scheier, M. F., e Carver, C. S. (1985).

Optimism, coping, and health: Assessment and implications of generalized outcome expectancies. Health Psychology, 4, 219-247.

Scheier, M. F., Carver, C. S. (1993). On the power of positive thinking: The benefits of being optimistic. Current Directions in Psychological Science, 2, 26-30.

Scheier, M. F., Carver, C. S. (1993). On the power of positive thinking: The benefits of being optimistic. Current Directions in Psychological Science, 2, 26-30.

Scheier, M. F., Carver, C. S. (1987). Dispositional optimism and physical well-being: The influence of generalized outcome expectancies on health. Journal of Personality. Special Issue: Personality and Physical Health, 55, 169-210.

Scheier, M. F., Carver, C. S., Bridges, M. W. (1994). Distinguishing optimism from neuroticism (and trait anxiety, selfmastery, and self-esteem): A reevaluation of the Life Orientation Test. Journal of Personality and Social Psychology, 67, 1063-1078. 
Scheier, M.F., Carver, C.S., Segerstrom, S.C. (2010). Optimism. Clinical Psychology Review, 30, 879-889.

Schweizer, K., Beck-Seyffer, A., Schneider, R. (1999). Cognitive bias of optimism and its influence on psychological well-being. Psychological Report, 84, 627-636.

Seligman, M., Csikszentmihalyi, M. (2000). Positive psychology: An introducion. American Psychological Association, 55, 5-14.

Seligman, M. E. P. (1998). Learned optimism: How to change your mind and your life. New York: Free Press.

Steger, M. F. (2013). Experiencing meaning in life: Optimal functioning at the nexus of well-being, psychopathology, and spirituality. In The human quest for meaning. Routledge (211-230).

Strassle, C. G., McKee, E. A., Plant, D. D. (1999). Optimism as an indicator of psychological health: Using psychological assessment wisely. Journal of Personality Assessment, 72, 265-276.

Vehling, S. et al. (2011). Global meaning and meaning-related life attitudes: exploring their role in predicting depression, anxiety, and demoralization in cancer patients. Supportive Care in Cancer, 19, 513-520.
Wood, A. M., Joseph, S. (2010). The absence of positive psychological (eudemonic) well-being as a risk factor for depression: A ten year cohort study. Journal of Affective Disorders, 122, 213-217.

World Health Organisation (2001). The world health report-Mental health: New understanding, new hope. Geneva: World Health Organisation.

Woyciekoski, C., Stenert, F., Hutz, C. S. (2012). Determinantes do bem-estar subjetivo. Psico-PUCRS, 43, 280-288.

Zhang, Y. et al. (2015). The relationship between impulsivity and internet addiction in Chinese college students: a moderated mediation analysis of meaning in life and self-esteem. PloS one, 10, 1-10.

Ziller, R. C. et al. (1969). Self-esteem: a self-social construct. Journal of Consulting and Clinical Psychology, 33, 84-95 